\title{
Fatal hemorrhage in a patient with advanced soft tissue sarcoma following radiation and pazopanib treatment: A case report
}

\author{
KENTA KAWASAKI $^{1}$, YASUO HAMAMOTO ${ }^{2}$, JUNICHI FUKADA ${ }^{3}$, MASAYUKI ADACHI $^{1}$, \\ HIKARU SASAKI ${ }^{4}$, HIROMASA TAKAISHI $^{2}$, KAZUNARI YOSHIDA $^{4}$ and TAKANORI KANAI ${ }^{1}$ \\ ${ }^{1}$ Division of Gastroenterology and Hepatology, Department of Internal Medicine; ${ }^{2}$ Keio Cancer Center; \\ Departments of ${ }^{3}$ Radiology and ${ }^{4}$ Neurosurgery, Keio University School of Medicine, Tokyo 160-8587, Japan
}

Received January 4, 2015; Accepted February 1, 2016

DOI: $10.3892 / 01.2016 .4246$

\begin{abstract}
A 71-year-old Japanese woman presented to the Department of Neurosurgery of Keio University School of Medicine (Tokyo, Japan) with a leiomyosarcoma originating in the infratemporal fossa and an 85-mm metastasis to the right lung. Since the pulmonary lesion was associated with risk of airway obstruction, radiation therapy (50 Gy/20 fractions) was administered to the patient, which resulted in remarkable tumor shrinkage. Subsequently, the patient was administered $800 \mathrm{mg} /$ day pazopanib for 10 weeks, which resulted in further reduction in tumor size. However, the patient succumbed to a massive hemorrhage of sudden onset. Three possible explanations for the fatal hemorrhage are: i) adverse reaction to pazopanib; ii) side effects of radiation; and iii) remarkable tumor shrinkage. As a result, it may be proposed that the synergic effects of the aforementioned hypotheses may have been responsible for the bleeding observed in the present case. Therefore, clinicians should be alert to the possibility of this adverse event. In the present study, the first case of mortality due to massive hemorrhage following remarkable tumor shrinkage induced by radiation therapy and subsequent pazopanib treatment is reported.
\end{abstract}

\section{Introduction}

Angiogenesis is important in tumor progression, and has been one of the targets of cancer treatment (1). Angiogenesis inhibitors have been demonstrated to confer survival benefits in patients with colorectal, non-small cell lung, breast and renal cell carcinoma (2). Several angiogenesis inhibitors are currently available worldwide, and unique adverse events associated with their use, which do not occur with

Correspondence to: Dr Hiromasa Takaishi, Keio Cancer Center, Keio University School of Medicine, 35 Shinanomachi, Shinjuku-ku, Tokyo 160-8587, Japan

E-mail: takaishi@z6.keio.jp

Key words: leiomyosarcoma, pazopanib, adverse event, hemorrhage, post-radiation therapy traditional chemotherapy regimens, have been suspected (3). These include modest-to-mild hypertension, proteinuria, and epistaxis, which are manageable, although serious adverse events such as gastrointestinal hemorrhage, gastrointestinal tract perforation, and arterial and venous thromboembolisms have also been reported (2).

The oral tyrosine kinase inhibitor pazopanib was the first molecular-targeted agent approved for the treatment of advanced soft tissue sarcoma (4). Pazopanib is a novel multi-kinase inhibitor that inhibits angiogenesis, and has been demonstrated to improve progression-free survival, compared with placebo, in the PALETTE trial (4). The majority of the adverse events reported for this drug, including hypertension, fatigue and diarrhea, were mild-to-modest, although severe hemorrhage has also been reported (5-8).

Thus far, bleeding events associated with the use of bevacizumab, sorafenib, sunitinib and pazopanib have been reported $(9,10)$. To avoid them, care must be taken when selecting patients for angiogenesis treatments (10).

In the present study, a case of mortality due to massive hemorrhage following remarkable tumor shrinkage induced by radiation therapy and subsequent pazopanib treatment is reported.

\section{Case report}

A 71-year-old Japanese woman was referred to the Department of Neurosurgery of Keio University School of Medicine (Tokyo, Japan) in September 2007 for a left buccal palsy. The patient had never smoked and did not drink alcohol. Her medical history revealed hypertension, which was well-controlled with candesartan ( $8 \mathrm{mg} / \mathrm{day}$ ), and chronic hepatitis $\mathrm{C}$, which was inactive. Her performance status was evaluated as 1 , due to hearing impairment. Written informed consent was obtained from the patient's guardian for the publication of the study.

An infratemporal fossa tumor was diagnosed and removed surgically. The pathological diagnosis was leiomyosarcoma. In total, the patient underwent six attempts of surgical removal and three courses of radiotherapy (64 Gy/32 fractions, $54 \mathrm{~Gy} / 27$ fractions and $60 \mathrm{~Gy} / 30$ fractions; Clinac ${ }^{\circledR}$ iX System Linear Accelerator; Varian Medical Systems, Palo Alto, CA, USA) for local recurrence. Metastasis to the right lung was later identified, with rapidly progressing local invasion from the left 
infratemporal fossa into the maxillary sinus and oral cavity. In consequence, the patient underwent four cycles of systemic chemotherapy over three months with mesna $(1500 \mathrm{mg}$; intravenous), adriamycin (60 $\mathrm{mg} / \mathrm{m}^{2}$; intravenous), ifosfamide (2.5 g/ $\mathrm{m}^{2}$; intravenous) and dacarbazine $\left(300 \mathrm{mg} / \mathrm{m}^{2}\right.$; intravenous) (MAID) regimen. Remarkable responses were achieved in the locally advanced primary tumors and pulmonary lesions. Following 2 years of treatment, although the primary lesion had stabilized, the lung metastasis had progressed as revealed by a computed tomography scan (Fig. 1A; Aquilion ${ }^{\mathrm{TM}}$; Toshiba Medical Systems Ltd., Otawara, Japan). A further course of MAID chemotherapy was administered. However, the tumor was refractory to this treatment, and the patient was referred to the Keio Cancer Center of Keio University School of Medicine (Tokyo, Japan) in January 2013. Since her pulmonary lesion was increasing in size, thus increasing the risk of airway obstruction, radiation therapy $\left(50 \mathrm{~Gy} / 20\right.$ fractions; Clinac ${ }^{\circledR}$ iX System Linear Accelerator) was administered to the patient, which resulted in $13 \%$ tumor shrinkage (Fig. 1B). Subsequently, the patient was administered oral pazopanib daily (800 mg/day; GlaxoSmithKline, Brentford, UK) as palliative chemotherapy in the outpatient clinic.

Upon initiation of pazopanib, grade 2 hypertension and grade 2 mucositis [according to the National Cancer Institute Common Terminology Criteria for Adverse Events (11)] were observed on day 14. Due to the development of corns on both feet on day 55 (known as grade 3 palmar-plantar erythrodysesthesia syndrome), pazopanib was ceased for 2 weeks, and recommenced when this symptom had resolved. At this stage, $38 \%$ shrinkage of the tumor in the right lung was evident (Fig. 1C). However, following 3 months of treatment, the patient suddenly vomited blood at home and succumbed to the hemorrhage.

At autopsy, the primary leiomyosarcoma in the brain and the metastasis in the right lung were confirmed. However, no ulcer formation in the gastrointestinal tract, ruptured blood vessel or cavity formation were observed.

\section{Discussion}

The present study reports a case of hemorrhage-associated mortality and tumor shrinkage induced by radiation therapy, followed by pazopanib treatment. There are three main possible explanations for the fatal hemorrhage.

First, the possibility that the hemorrhage was an adverse reaction to pazopanib must be considered, since vascular endothelial growth factor (VEGF) and multi-kinase inhibitors may cause hemorrhage, and pazopanib has been reported to cause gastrointestinal and intracranial hemorrhage (5-8).

Second, the effects of radiation continue for few months post-treatment and include severe fibrosis (3). A previous study reported one patient who succumbed to hemorrhage upon receiving $>50$ Gy of radiation to the pulmonary artery and bronchus (12). Furthermore, another report warned against using stereotactic body radiation therapy in patients with tumors located near the central airways due to various adverse effects, including hemorrhagic mortality (13). In the present case, although the patient was exposed to $<50 \mathrm{~Gy}$ dose, the radiation may still have been responsible for the hemorrhage.
A

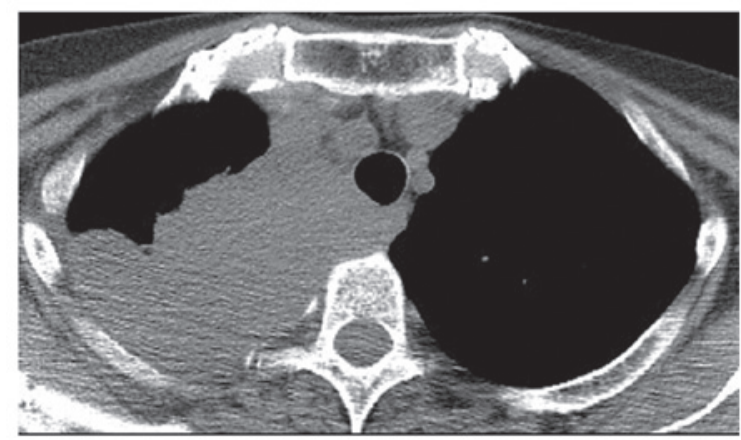

B

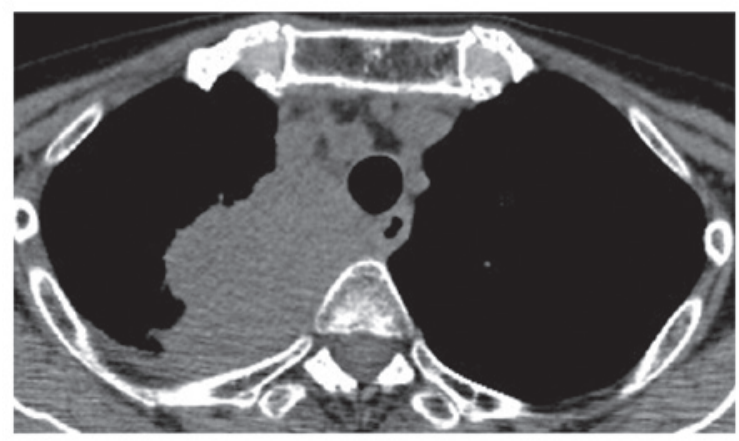

C

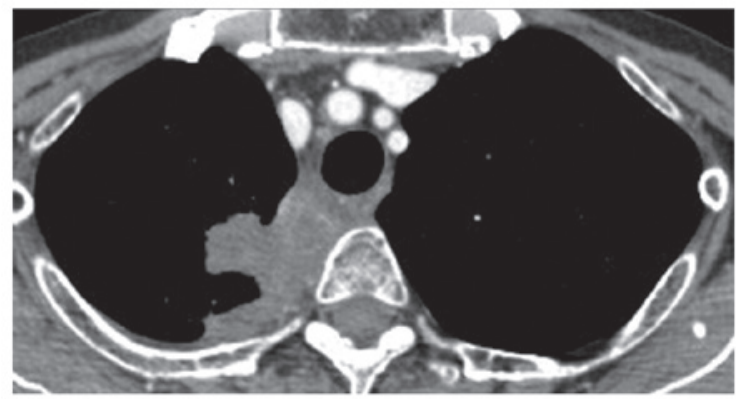

Figure 1. (A) Lung metastasis pre-radiation. CT scan image revealed an 85-mm metastatic lesion in the right lung. (B) Lung metastasis post-radiation. CT scan image demonstrated a $13 \%$ reduction in size of the metastatic lesion in the right lung following radiation treatment with $50 \mathrm{~Gy} / 20 \mathrm{frac}-$ tions. (C) Lung metastasis post-pazopanib treatment. CT scan image revealed a $38 \%$ reduction in size of the metastatic lesion in the right lung following radiation and subsequent pazopanib treatment. CT, computed tomography.

Third, there is a risk of severe bleeding in patients with remarkable shrinkage of tumor following irradiation, which is generally associated with fibrosis in the surrounding tissues (3). As reported by Hui et al (3), tumor necrosis and cavitation are associated with bleeding events. Thus, tumor shrinkage following treatment with anti-angiogenic therapy is associated with an increased risk of bleeding (3). In the present case, although there was no cavity formation, remarkable tumor shrinkage had occurred. Considering that the lung metastasis in the present patient was adjacent to the trachea, shrinkage of the tumor may have caused perforation of the trachea and subsequent hemorrhage from the tumor.

Therefore, it may be hypothesized that the synergic effects of pazopanib, radiotherapy and tumor shrinkage may have been responsible for the bleeding in the present case.

In conclusion, remarkable tumor response associated with radiation therapy may be a risk factor for severe hemorrhage when combined with pazopanib. Thus, clinicians should be 
alert to the possibility of this adverse event. In this situation, delaying the introduction of pazopanib therapy may be an option. Further investigation of the possible mechanisms leading to fatal hemorrhage, and analysis of the risk factors associated with the hemorrhagic adverse events caused by VEGF and multi-kinase inhibitors, are required.

\section{References}

1. Ferrara N: Role of vascular endothelial growth factor in physiologic and pathologic angiogenesis: Therapeutic implications. Semin Oncol 29 (Suppl 16): S10-S14, 2002.

2. Hapani S, Sher A, Chu D and Wu S: Increased risk of serious hemorrhage with bevacizumab in cancer patients: A meta-analysis. Oncology 79: 27-38, 2010.

3. Hui EP, Ma BB, King AD, Mo F, Chan SL, Kam MK, Loong HH, Ahuja AT, Zee BC and Chan AT: Hemorrhagic complications in a phase II study of sunitinib in patients of nasopharyngeal carcinoma who has previously received high-dose radiation. Ann Oncol 22: 1280-1287, 2011.

4. van der Graaf WT, Blay JY, Chawla SP, Kim DW, Bui-Nguyen B, Casali PG, Schöffski P, Aglietta M, Staddon AP, Beppu Y, et al; EORTC Soft Tissue and Bone Sarcoma Group; PALETTE study group: Pazopanib for metastatic soft-tissue sarcoma (PALETTE) A randomised, double-blind, placebo-controlled phase 3 trial. Lancet 379: 1879-1886, 2012

5. Iwamoto FM, Lamborn KR, Robins HI, Mehta MP, Chang SM, Butowski NA, Deangelis LM, Abrey LE, Zhang WT, Prados MD and Fine HA: Phase II trial of pazopanib (GW786034), an oral multi-targeted angiogenesis inhibitor, for adults with recurrent glioblastoma (North American Brain Tumor Consortium Study 06-02). Neuro Oncol 12: 855-861, 2010.

6. Taylor SK, Chia S, Dent S, Clemons M, Agulnik M, Grenci P, Wang L, Oza AM, Ivy P, Pritchard KI and Leighl NB: A phase II study of pazopanib in patients with recurrent or metastatic invasive breast carcinoma: A trial of the Princess Margaret Hospital phase II consortium. Oncologist 15: 810-818, 2010.
7. Bible KC, Suman VJ, Menefee ME, Smallridge RC, Molina JR, Maples WJ, Karlin NJ, Traynor AM, Kumar P, Goh BC, et al; Mayo Phase 2 Consortium; Mayo Clinic Endocrine Malignances Disease Oriented Group: A multiinstitutional phase 2 trial of pazopanib monotherapy in advanced anaplastic thyroid cancer. J Clin Endocrinol Metab 97: 3179-3184, 2012.

8. Glade Bender JL, Lee A, Reid JM, Baruchel S, Roberts T, Voss SD, Wu B, Ahern CH, Ingle AM, Harris P, et al: Phase I pharmacokinetic and pharmacodynamic study of pazopanib in children with soft tissue sarcoma and other refractory solid tumors: A children's oncology group phase I consortium report. J Clin Oncol 31: 3034-3043, 2013.

9. Hang XF, Xu WS, Wang JX, Wang L, Xin HG, Zhang RQ and Ni W: Risk of high-grade bleeding in patients with cancer treated with bevacizumab: A meta-analysis of randomized controlled trials. Eur J Clin Pharmacol 67: 613-623, 2011.

10. Schutz FA, Je Y, Richards CJ and Choueiri TK: Meta-analysis of randomized controlled trials for the incidence and risk of treatment-related mortality in patients with cancer treated with vascular endothelial growth factor tyrosine kinase inhibitors. J Clin Oncol 30: 871-877, 2012.

11. National Cancer Institute: Common terminology criteria for adverse events, version 4.0.3. evs.nci.nih.gov/ftp1/CTCAE/ CTCAE_4.03_2010-06-14_QuickReference_5x7.pdf. Accessed Feb 6, 2016.

12. Nishimura S, Takeda A, Sanuki N, Ishikura S, Oku Y, Aoki Y, Kunieda E and Shigematsu N: Toxicities of organs at risk in the mediastinal and hilar regions following stereotactic body radiotherapy for centrally located lung tumors. J Thorac Oncol 9: 1370-1376, 2014

13. Timmerman R, McGarry R, Yiannoutsos C, Papiez L, Tudor K, DeLuca J, Ewing M, Abdulrahman R, DesRosiers C, Williams M and Fletcher J: Excessive toxicity when treating central tumors in a phase II study of stereotactic body radiation therapy for medically inoperable early-stage lung cancer. J Clin Oncol 24: 4833-4839, 2006. 